\title{
Effects of meteorological factors on epidemic malaria in Ethiopia: a statistical modelling approach based on theoretical reasoning
}

\author{
T. A. ABEKU ${ }^{1,2 *}$, S. J. DE VLAS ${ }^{1}$, G. J. J. M. BORSBOOM ${ }^{1}$, A. TADEGE ${ }^{3}$, Y. GEBREYESUS ${ }^{3}$, \\ H. GEBREYOHANNES ${ }^{3}$, D. ALAMIREW ${ }^{4}$, A. SEIFU ${ }^{4}$, N. J. D. NAGELKERKE ${ }^{\mathbf{1 , 5}}$ \\ and J. D. F. HABBEMA ${ }^{1}$ \\ ${ }^{1}$ Department of Public Health, Erasmus MC, University Medical Center Rotterdam, The Netherlands \\ ${ }^{2}$ Disease Control and Vector Biology Unit, Department of Infectious and Tropical Diseases, London School of Hygiene \\ and Tropical Medicine, UK \\ ${ }^{3}$ National Meteorological Services Agency, Addis Ababa, Ethiopia \\ ${ }^{4}$ Disease Prevention and Control Department, Ministry of Health, Addis Ababa, Ethiopia \\ ${ }^{5}$ Department of Medical Statistics, Leiden University Medical Center, The Netherlands
}

(Received 10 September 2003; revised 21 November 2003; accepted 26 November 2003)

\section{SUMMARY}

This study was conducted to quantify the association between meteorological variables and incidence of Plasmodium falciparum in areas with unstable malaria transmission in Ethiopia. We used morbidity data pertaining to microscopically confirmed cases reported from 35 sites throughout Ethiopia over a period of approximately 6-7 years. A model was developed reflecting biological relationships between meteorological and morbidity variables. A model that included rainfall 2 and 3 months earlier, mean minimum temperature of the previous month and $P$. falciparum case incidence during the previous month was fitted to morbidity data from the various areas. The model produced similar percentages of overestimation (19.7\% of predictions exceeded twice the observed values) and under-estimation (18.6\% were less than half the observed values). Inclusion of maximum temperature did not improve the model. The model performed better in areas with relatively high or low incidence ( $>85 \%$ of the total variance explained) than those with moderate incidence $(55-85 \%$ of the total variance explained). The study indicated that a dynamic immunity mechanism is needed in a prediction model. The potential usefulness and drawbacks of the modelling approach in studying the weather-malaria relationship are discussed, including a need for mechanisms that can adequately handle temporal variations in immunity to malaria.

Key words: malaria, Plasmodium falciparum, highland, epidemic, climate, Ethiopia, linear mixed model.

\section{INTRODUCTION}

Epidemic malaria remains a major public health concern in highland and arid areas in tropical countries (Lindsay \& Martens, 1998; Nájera et al. 1998). Changes in weather conditions have probably played a major role as the cause of most of the severe epidemics. Increased temperature in cooler environments shortens the parasite's life-cycle within the vector, thus increasing transmission probability before the mosquito vector dies (MacDonald, 1957; Molineaux, 1988). Increased temperature would also increase the rate of mosquito emergence from breeding places, and in the presence of rainfall increased humidity results in longer survival of the vector to transmit the parasite (Hay et al. 2000).

* Corresponding author: Disease Control and Vector Biology Unit, Department of Infectious and Tropical Diseases, London School of Hygiene and Tropical Medicine, Keppel Street, London WC1E 7HT, UK. Tel: +44 (0) 207612 7861. Fax: +44 (0) 207580 9075. E-mail: tarekegn.abeku@1shtm.ac.uk
Rainfall also affects the abundance of mosquito breeding sites.

In the Ethiopian highlands, several large-scale epidemics have been reported since the 1950s. In 1958, an estimated 150000 people died during a widespread epidemic of malaria in the highlands (Fontaine et al. 1961). Several epidemics have been reported since then. Abnormal transmission of unusual proportions has affected the highlands and highland-fringe areas in 1988 and 1991-92 which was associated with abnormally increased minimum temperature (Abeku et al. 2003). More recently, epidemics have occurred in the highlands during the second half of the last decade; in particular a widespread epidemic in 1998 was largely attributed to an El Niño event (unpublished data). The association of abnormal weather conditions and increased malaria incidence has been reported in several studies. In the Punjab province of India, epidemics were shown to be significantly more prevalent in a year with a wet (high) monsoon rainfall following a dry El Niño year than in other years, while in Sri Lanka, epidemics were significantly more prevalent during El Niño 
years, when the same south-west monsoon tends to fail (Bouma \& Van der Kaay, 1996). In Venezuela, malaria significantly increased in the year following an El Niño event (Bouma \& Dye, 1997).

Currently there is a need for systems for epidemic early warning in areas at risk (Myers et al. 2000; Thomson \& Connor, 2001). Previously, we have demonstrated that incidence in areas with unstable transmission may not be predicted well from historical morbidity patterns alone even when a statistically more sophisticated ARIMA (autoregressive and integrated moving average) method is used (Abeku et al. 2002). In areas with highly variable transmission, the use of predictor variables such as temperature and rainfall together with past patterns of incidence might lead to more accurate predictions.

The aim of this study was to quantify the effects of meteorological factors on malaria incidence in areas with unstable transmission using a statistical model based on theoretical reasoning. On the basis of biological arguments, we derived a linear mixed model for monthly data including rainfall, temperature and incidence of confirmed Plasmodium falciparum cases reported from 35 sites across Ethiopia. We also tested whether extending the model by including more variables would significantly improve the basic model. Moreover, we compared the performance of the basic model with methods that use historical morbidity patterns for studying the impact of weather variables on incidence after one month interval.

\section{MATERIALS AND METHODS}

\section{Data used for analysis}

We used malaria morbidity data (microscopically confirmed cases) reported from 35 Sector Malaria Control Offices (SMCOs) throughout Ethiopia between September 1986 and August 1993. A sector is an area delineated for the purpose of malaria control and covers 2-5 districts, each with approximately 75000 to 150000 inhabitants. The malaria cases were seen at Malaria Detection and Treatment Posts (MDTPs) located in catchment areas of SMCOs, which are supposed to report to their respective SMCOs every month. We assumed that among the confirmed cases reported monthly, the majority were diagnosed at malaria laboratories which were based at the SMCOs. Most of the other MDTPs (e.g. health centres, hospitals, etc.) irregularly report to SMCOs and when they do, the reports constitute only a small proportion of the total confirmed cases in each sector. Furthermore, in view of the limited modes of transportation in rural areas, it is very likely that most of the malaria cases seen at a sector's malaria laboratory originated from localities not far away from the base town of the sector.
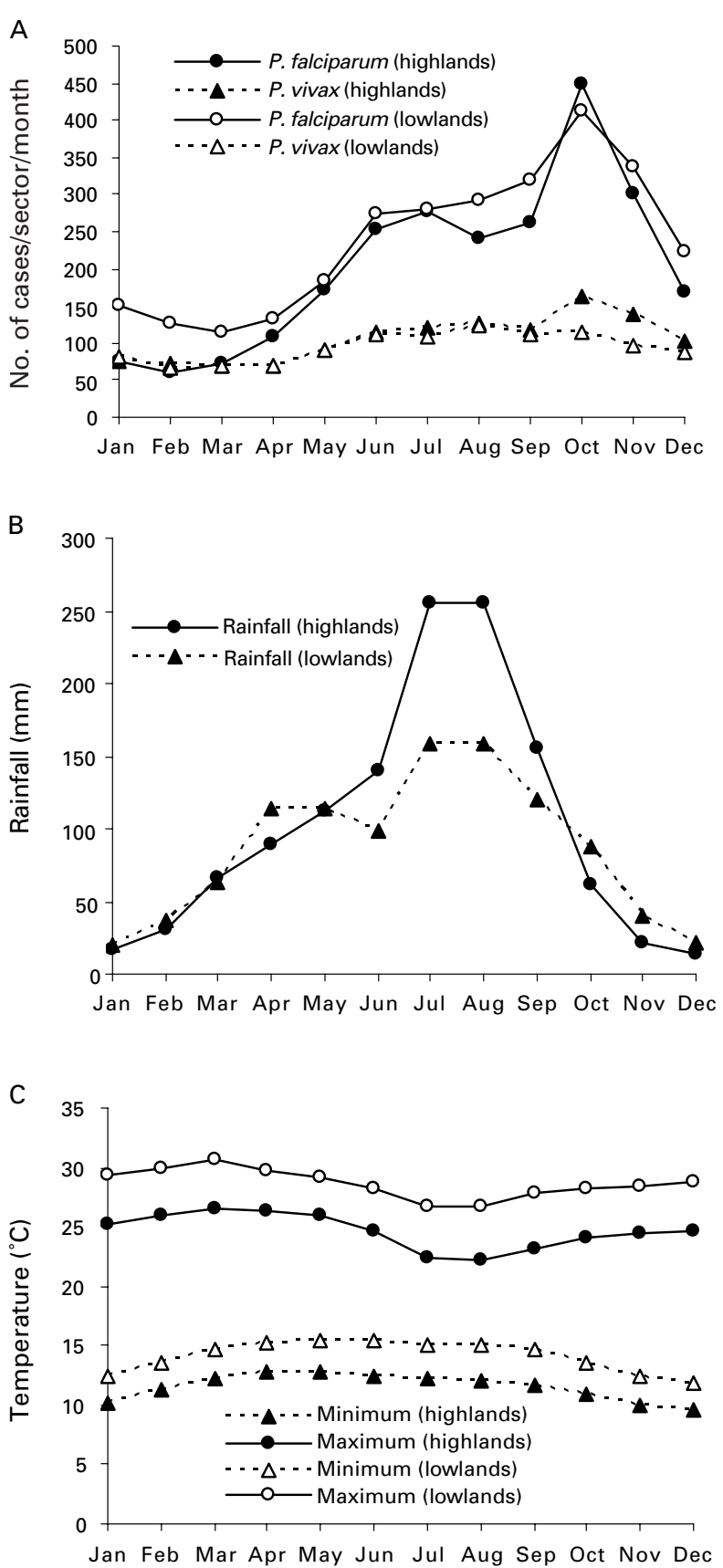

Fig. 1. Seasonal variations in (A) incidence of Plasmodium falciparum and P. vivax malaria, (B) rainfall and

(C) temperature, in 'highlands' (>1750 m) and 'lowlands' $(\leqslant 1750 \mathrm{~m})$ in Ethiopia.

During September 1986-August 1993, on average 320 confirmed malaria cases were reported per sector per month. $P$. falciparum and $P$. vivax constituted $68.7 \%$ and $31 \cdot 3 \%$ of the total 604589 malariapositive cases, respectively. To study the seasonal pattern of malaria at different altitudes, the sectors were grouped as 'highlands' (above $1750 \mathrm{~m}, n=18$ ) and 'lowlands' (1750 $\mathrm{m}$ and below, $n=17)$. Both groups have a similar seasonal pattern of incidence (Fig. 1A) with a peak in $P$. falciparum incidence in October ( $P$. vivax showed much less interseasonal variation). The high degree of seasonality of 
Table 1. Characteristics of the 35 study areas (sectors) and area-specific random effects of the basic linear mixed model fitted to log-transformed Plasmodium falciparum incidence data reported during September 1986-August 1993

\begin{tabular}{|c|c|c|c|c|c|c|c|}
\hline \multirow[b]{2}{*}{ Sector } & \multirow{2}{*}{$\begin{array}{l}\text { Altitude } \\
\text { (m) }\end{array}$} & \multicolumn{2}{|c|}{$\begin{array}{l}\text { Average daily } \\
\text { temperature }\left({ }^{\circ} \mathrm{C}\right)\end{array}$} & \multirow{2}{*}{$\begin{array}{l}\text { Annual } \\
\text { rainfall } \\
(\mathrm{mm})\end{array}$} & \multirow{2}{*}{$\begin{array}{l}\text { Mean monthly } \\
\text { no. of falciparum } \\
\text { malaria cases }\end{array}$} & \multirow[b]{2}{*}{ Intercept } & \multirow{2}{*}{$\begin{array}{l}\text { Coefficient of } \\
\log _{\mathrm{e}} \text { (incidence) ir } \\
\text { previous month }\end{array}$} \\
\hline & & Minimum & Maximum & & & & \\
\hline Abomsa & 1800 & $15 \cdot 3$ & $28 \cdot 2$ & 960 & 706 & -0.92 & $0 \cdot 78$ \\
\hline Adiszemen & 1550 & $10 \cdot 9$ & $28 \cdot 3$ & 1315 & 63 & $-0 \cdot 91$ & $0 \cdot 71$ \\
\hline Alaba & 1750 & $11 \cdot 2$ & $26 \cdot 9$ & 1032 & 252 & $-0 \cdot 71$ & $0 \cdot 82$ \\
\hline Alamata & 1580 & $14 \cdot 8$ & $29 \cdot 9$ & 754 & 302 & $-1 \cdot 13$ & $0 \cdot 77$ \\
\hline Alemketema & 2280 & $12 \cdot 6$ & $24 \cdot 8$ & 1174 & 30 & $-1 \cdot 18$ & $0 \cdot 68$ \\
\hline Ambo & 2130 & $11 \cdot 4$ & $25 \cdot 2$ & 1024 & 6 & $-1 \cdot 36$ & $0 \cdot 57$ \\
\hline Arba Minch & 1290 & $15 \cdot 1$ & $29 \cdot 9$ & 839 & 569 & -0.92 & $0 \cdot 75$ \\
\hline Asela & 2350 & $8 \cdot 5$ & $21 \cdot 0$ & 1208 & 264 & -0.79 & $0 \cdot 76$ \\
\hline Awasa & 1750 & $12 \cdot 2$ & $26 \cdot 7$ & 953 & 101 & $-1 \cdot 05$ & $0 \cdot 69$ \\
\hline Bahirdar & 1770 & $11 \cdot 5$ & $26 \cdot 6$ & 1466 & 263 & -0.79 & $0 \cdot 76$ \\
\hline Bati & 1660 & $13 \cdot 0$ & $28 \cdot 3$ & 873 & 382 & $-1 \cdot 12$ & $0 \cdot 80$ \\
\hline Bedele & 2030 & $12 \cdot 0$ & $25 \cdot 2$ & 1793 & 12 & $-1 \cdot 20$ & $0 \cdot 50$ \\
\hline Chagni & 1620 & $12 \cdot 3$ & $27 \cdot 7$ & 1762 & 177 & $-0 \cdot 96$ & $0 \cdot 75$ \\
\hline Debretabor & 2690 & $9 \cdot 4$ & $22 \cdot 2$ & 1565 & 65 & $-0 \cdot 83$ & $0 \cdot 70$ \\
\hline Debrezeit & 1900 & $11 \cdot 5$ & $26 \cdot 2$ & 861 & 260 & $-1 \cdot 00$ & $0 \cdot 79$ \\
\hline Dembidolo & 1850 & $13 \cdot 0$ & $25 \cdot 0$ & 1225 & 98 & $-0 \cdot 81$ & $0 \cdot 64$ \\
\hline Dila & 1500 & $11 \cdot 3$ & $27 \cdot 8$ & 1323 & 26 & $-1 \cdot 01$ & $0 \cdot 67$ \\
\hline Fiche & 2750 & $7 \cdot 7$ & $20 \cdot 2$ & 1211 & 40 & $-0 \cdot 87$ & $0 \cdot 70$ \\
\hline Finoteselam & 1900 & $11 \cdot 8$ & $27 \cdot 3$ & 1129 & 96 & $-1 \cdot 11$ & $0 \cdot 75$ \\
\hline Gambela & 480 & $18 \cdot 6$ & $35 \cdot 8$ & 1228 & 609 & $-1 \cdot 02$ & $0 \cdot 76$ \\
\hline Gode & 295 & $22 \cdot 5$ & $34 \cdot 8$ & 262 & 44 & $-1 \cdot 00$ & $0 \cdot 57$ \\
\hline Harer & 2100 & $13 \cdot 5$ & $25 \cdot 2$ & 713 & 276 & $-1 \cdot 38$ & $0 \cdot 84$ \\
\hline Hirna & 2050 & $12 \cdot 2$ & $25 \cdot 6$ & 1041 & 14 & $-1 \cdot 21$ & $0 \cdot 69$ \\
\hline Hosana & 2200 & $10 \cdot 4$ & $22 \cdot 5$ & 1186 & 37 & $-0 \cdot 92$ & $0 \cdot 67$ \\
\hline Jima & 1725 & $11 \cdot 1$ & $26 \cdot 9$ & 1506 & 20 & $-1 \cdot 13$ & $0 \cdot 67$ \\
\hline Jinka & 1480 & $15 \cdot 7$ & $27 \cdot 0$ & 1268 & 70 & $-1 \cdot 15$ & $0 \cdot 68$ \\
\hline Kombolcha & 1903 & $11 \cdot 8$ & $25 \cdot 9$ & 1049 & 663 & $-1 \cdot 33$ & $0 \cdot 86$ \\
\hline Metehara & 960 & $17 \cdot 5$ & $32 \cdot 7$ & 543 & 544 & $-0 \cdot 82$ & $0 \cdot 73$ \\
\hline Mizanteferi & 1370 & $15 \cdot 5$ & $27 \cdot 4$ & 2180 & 44 & $-1 \cdot 54$ & $0 \cdot 70$ \\
\hline Nazret & 1622 & $14 \cdot 0$ & $27 \cdot 9$ & 861 & 291 & $-0 \cdot 95$ & $0 \cdot 75$ \\
\hline Negele & 1544 & $13 \cdot 2$ & $25 \cdot 8$ & 764 & 24 & $-1 \cdot 19$ & $0 \cdot 65$ \\
\hline Nekemte & 2080 & $12 \cdot 2$ & $23 \cdot 7$ & 2089 & 130 & $-0 \cdot 98$ & $0 \cdot 71$ \\
\hline Sodo & 2020 & $13 \cdot 1$ & $24 \cdot 3$ & 1263 & 370 & $-1 \cdot 20$ & $0 \cdot 81$ \\
\hline Weliso & 2000 & $11 \cdot 8$ & $24 \cdot 8$ & 1203 & 106 & $-1 \cdot 05$ & $0 \cdot 74$ \\
\hline Zway & 1640 & $13 \cdot 5$ & $26 \cdot 4$ & 757 & 426 & $-1 \cdot 01$ & $0 \cdot 78$ \\
\hline
\end{tabular}

falciparum malaria is closely associated with seasonal variation in rainfall and temperature. Weather data between January 1950 and December 1998 (monthly rainfall, and minimum and maximum temperatures) and altitudes of base towns of the SMCOs were obtained from the National Meteorological Services Agency of Ethiopia (Table 1). In most areas, the main rainy season is between June and September with peak rains falling in July and August (Fig. 1B). On average, the highland sectors received more rainfall than did the lowlands. Average daily temperature in the highlands ranged from $17 \cdot{ }^{\circ} \mathrm{C}$ in December to $19 \cdot 5{ }^{\circ} \mathrm{C}$ in April, whereas in the lowlands, it ranged from $20.7{ }^{\circ} \mathrm{C}$ in December to $30.6{ }^{\circ} \mathrm{C}$ in March. Mean monthly minimum and maximum temperatures differed (as expected) between highlands and lowlands (Fig. 1C). Minimum and maximum temperatures also show different patterns of seasonal variation. During the rainy months, maximum temperature declines while minimum temperature remains unchanged. After September, minimum temperature gradually falls to a minimum value in December, while, in contrast, maximum temperature increased after September to a peak in March.

\section{Data transformation and imputation}

To obtain approximate Normal distribution, $\log$ and cube-root transformations were applied to incidence and rainfall data, respectively. Monthly minimum and maximum temperature data were assumed to have Normal distribution. Prior to log transformation, a value of 1 was added to all monthly number of cases to avoid transformation problem which arises in the case of 0 values.

Before fitting models, missing values of rainfall and temperature were imputed using linear interpolation (for gaps of up to 5 months) or by taking 
seasonal average values (for gaps of more than 5 months). The value of a missing data point for month $t$ (i.e. $X_{t}$ ) was estimated as:

$$
X_{t}=\left\{\begin{array}{lr}
\bar{X}_{t}+X_{t-m}-\bar{X}_{t-m}+\frac{m\left(X_{t+n}-\bar{X}_{t+n}-X_{t-m}+\bar{X}_{t-m}\right)}{m+n} & \text { if } m+n \leqslant 5 \\
\bar{X}_{t} & \text { otherwise, }
\end{array}\right.
$$

where $\bar{X}_{t}$ is the seasonal average (of the transformed series) for the corresponding calendar month, $m$ is the number of missing observations from the last observed value up to time $t$ and $n$ is the lead time to the next observed value in the 'future'. Of the data points relevant to the basic model (described below), the percentage imputed values of rainfall and minimum temperature were $12.7 \%$ and $15 \cdot 8 \%$, respectively.

\section{Theoretical reasoning}

In areas with low malaria endemicity, the number of new malaria cases in month $t$ (denoted as $I_{t}$ ) can be considered to depend on the number of new cases in the previous month multiplied by the vectorial capacity during the previous month (vectorial capacity is defined as the average number of secondary malaria cases potentially disseminated in a susceptible population by vector mosquitoes per day from a single primary case). This is due to the fact that nearly all newly infected individuals develop clinical illness as a result of lack of immunity. In areas with high endemicity, many people are (partially) immune and (mostly) not clinically ill, but still infectious; only people who lack sufficient immunity would visit health facilities for treatment, and this means that the number of new cases is mainly determined by vectorial capacity in the previous month alone. The minimum generation time (sometimes referred to as the 'incubation interval - i.e. the duration of a complete gametocyte-to-gametocyte cycle or the time from take-up of gametocytes by the vector until production of gametocytes by the next host after transmission), normally has a length of approximately 1 month in tropical temperatures, and this corresponds to the monthly character of the data used for analysis. These considerations can be generalized in the following equation:

$I_{t}=a I_{t-1}^{b} C_{t-1}$,

where $a$ and $b$ are area-specific constants, and $C_{t-1}$ is vectorial capacity in month $t-1$. If $b$ is (close to) 0 , we have $I_{t}=a C_{t-1}$ as expected in areas with high endemicity. On the other hand, if $b$ is (close to) 1 , we have $I_{t}=a I_{t-1} C_{t-1}$ as expected in areas with low endemicity.

$C_{t}$ is defined as the product of mosquito density in relation to the human population $\left(M_{t}\right)$ and vectorial capacity per mosquito $\left(W_{t}\right)$ in month $t$ :

$C_{t}=M_{t} W_{t}$.

We assume $M_{t}$ depends on rainfall during the previous 2 months and some area-specific constant $M$, and that there are enough mosquitoes present to generate an infinite number of offspring, whereby the presence of breeding sites is the limiting factor. Rainfall will be represented as amount during a particular month relative to average annual total for each area. Our assumption for taking rainfall relative to the annual total was that absolute rainfall is not so important but the consequences of rainfall in terms of number (and duration) of mosquito breeding sites are important. These consequences differ strongly among areas (depending on vegetation, soil type, presence of rivers, topography etc.) and therefore there cannot be an absolute relationship between rainfall and vector abundance. For example, if absolute rainfall would be used then a doubling of rainfall in a relatively dry area would have relatively little impact, as the difference involved is small. Thus, the effects of this doubling would be underestimated. In a very wet area, these effects would be overestimated. Thus we have:

$M_{t}=M \exp \left(\beta_{1} \frac{R_{t-1}}{R}+\beta_{2} \frac{R_{t-2}}{R}\right)$,

where $R_{t-1}$ and $R_{t-2}$ denote rainfall in months $t-1$ and $t-2$, respectively, $R$ is an area-specific average annual rainfall, and $\beta_{1}$ and $\beta_{2}$ are statistical coefficients of rainfall relative to annual total in months $t-1$ and $t-2$, respectively, to be estimated from data.

Vectorial capacity per mosquito $W_{t}$ in eqn (3) was assumed to depend to a large extent on temperature, and was represented by the sum of a linear and a quadratic term of average minimum temperature $(T)$ after a preliminary exploration of the likely effect of temperature; hence, we can write:

$W_{t}=\exp \left(\beta_{3} T_{t}+\beta_{4} T_{t}^{2}\right)$,

where $\beta_{3}$ and $\beta_{4}$ are statistical coefficients of $T_{t}$ and $T_{t}^{2}$, respectively, to be estimated from data.

At higher temperatures, the sporogonic cycle of the malaria parasite within the mosquito would be shortened, increasing the probability of transmission (as the parasite would be more likely to be transmitted before the mosquito vector dies when the duration of the cycle is shortened). Temperature also has an effect on the length of the aquatic cycle of the mosquito, but in the present model, the effect on the parasite has been emphasized (and thus $M_{t}$ is assumed to depend entirely on rainfall as described in eqn (4)). In this regard, the effect of rainfall (a factor for mosquito production) was also made to precede that of temperature (a factor that acts on the parasite prior to transmission). 
Table 2. Estimates of the fixed parameters of the basic linear mixed model fitted to log-transformed Plasmodium falciparum incidence data reported from 35 sectors in different parts of Ethiopia during September 1986-August 1993

\begin{tabular}{lcc}
\hline \hline Effect & Estimate (s.E.) & $P$ \\
\hline Mean intercept $(\alpha)$ & $-1 \cdot 04(0 \cdot 22)$ & $<0 \cdot 0001$ \\
Mean log $(P$. falciparum incidence in previous month) $(b)$ & $0 \cdot 72(0 \cdot 02)$ & $<0 \cdot 0001$ \\
Rainfall relative to annual total 2 months earlier $\left(\beta_{1}\right)$ & $4 \cdot 12(0 \cdot 49)$ & $<0 \cdot 0001$ \\
Rainfall relative to annual total 3 months earlier $\left(\beta_{2}\right)$ & $0 \cdot 81(0 \cdot 49)$ & $0 \cdot 098$ \\
Minimum temperature in previous month $\left(\beta_{3}\right)$ & $0 \cdot 19(0 \cdot 03)$ & $<0 \cdot 0001$ \\
(Minimum temperature in previous month) squared $\left(\beta_{4}\right)$ & $-0 \cdot 0045(0 \cdot 0012)$ & $0 \cdot 0001$ \\
\hline \hline
\end{tabular}

After substitution we get:

$$
\begin{aligned}
I_{t}= & M a I_{t-1}^{b} \exp \left(\beta_{1} \frac{R_{t-2}}{R}+\beta_{2} \frac{R_{t-3}}{R}\right) \\
& \times \exp \left(\beta_{3} T_{t-1}+\beta_{4} T_{t-1}^{2}\right) .
\end{aligned}
$$

\section{Resulting statistical model}

After taking (natural) logarithms, eqn (6) can be rewritten as a linear mixed model for each sector $i$ as follows:

$$
\begin{aligned}
\log \left(I_{t, i}\right)= & \alpha_{i}+b_{i} \log \left(I_{t-1, i}\right)+\beta_{1} \frac{R_{t-2, i}}{R_{i}} \\
& +\beta_{2} \frac{R_{t-3, i}}{R_{i}}+\beta_{3} T_{t-1, i}+\beta_{4} T_{t-1, i}^{2}+\varepsilon_{t, i} .
\end{aligned}
$$

Here $\alpha_{i}=\log \left(M_{i}\right)+\log \left(a_{i}\right)$ denotes the sector-specific intercept, and $\varepsilon_{t, i}$ is a normally distributed random error with mean 0 and variance $\sigma^{2}$. This model describes the area-specific (log) incidence in month $t$ as a function of: (1) (log) incidence in the previous month; (2) rainfall 2 and 3 months earlier; and (3) average minimum temperature in the previous month. In eqn (7), between-sector differences in average incidence and in the effect of previous incidence were accounted for by the random (sector-specific) intercept $\alpha_{i}$ and the slope $b_{i}$ (i.e. parameter of previous incidence). The effects of rainfall and temperature were assumed identical across sectors. Using the MIXED procedure of the SAS/STAT ${ }^{\circledR}$ software of the SAS System Version 8.2 (SAS Institute Inc., Cary, NC 27513, USA), we estimated the intercept $\alpha_{i}$ and the slope $b_{i}$ of $\log \left(I_{t-1, i}\right)$ as sector-specific random effects, and $\beta_{1}, \beta_{2}, \beta_{3}$ and $\beta_{4}$, as fixed effects from the data (SAS Institute Inc., 1999; Verbeke \& Molenberghs, 2000). This procedure can handle problems related to spatial and temporal autocorrelations in the data set during estimation of model coefficients and their variance.

In order to judge the quality of the predictions, the model was also extended to include more meteorological variables at different lags. Likelihood ratio tests were performed to test the goodness-of-fit of the various extensions in comparison to the basic model given in eqn (7). Also, variance as explained was used to reflect the goodness-of-fit per sector. Predictions were considered not good enough if they exceeded twice the observed values (overestimation) or were less than half the observed values (under-estimation). Gross under-estimation in relation to missing epidemic events which was considered more important than over-estimation was also studied using a threshold value of 200 cases per month per sector, and the results were compared to other simpler models not using weather data, including a simple method using incidence of the previous month as a forecast value for the current month and a seasonal adjustment method that uses values of 3 previous months (Abeku et al. 2002).

\section{RESULTS}

The estimates of coefficients in the basic model represented by eqn (7), estimated from data from the 35 sectors, are given in Table 2. All included effects were statistically highly significant except rainfall 3 months earlier which was significant at the $10 \%$ level. Area-specific intercepts and incidence in the previous month are given in Table 1. The areaspecific effect of incidence in the previous month (i.e. term $b_{i}$ in model (7)) showed little variation between sectors, with a mean of 0.72 (95\% CI: $0 \cdot 69-0 \cdot 75)$. Three-quarters of the sectors had values of the coefficient between $0 \cdot 65$ and $0 \cdot 80$.

Observed and predicted values of the basic model are shown in Fig. 2. The model produced similar percentages of over-estimation $(19.7 \%)$ and underestimation (18.6\%). Especially high incidence values showed a good fit in the model. Detailed analysis of the under-estimation problem showed that about $10 \%$ of the observations were under-estimated by more than 200 cases per month, and about $5 \%$ were under-estimated by 400 cases per month. It was also found that sectors with normally high and low number of malaria cases had better fits than did sectors with moderate number of cases (Fig. 3). For most areas, the amount of variance in incidence explained by the model exceeded $80 \%$, and for nearly half of the 35 sectors this proportion exceeded $90 \%$. The model performed better in areas with relatively 


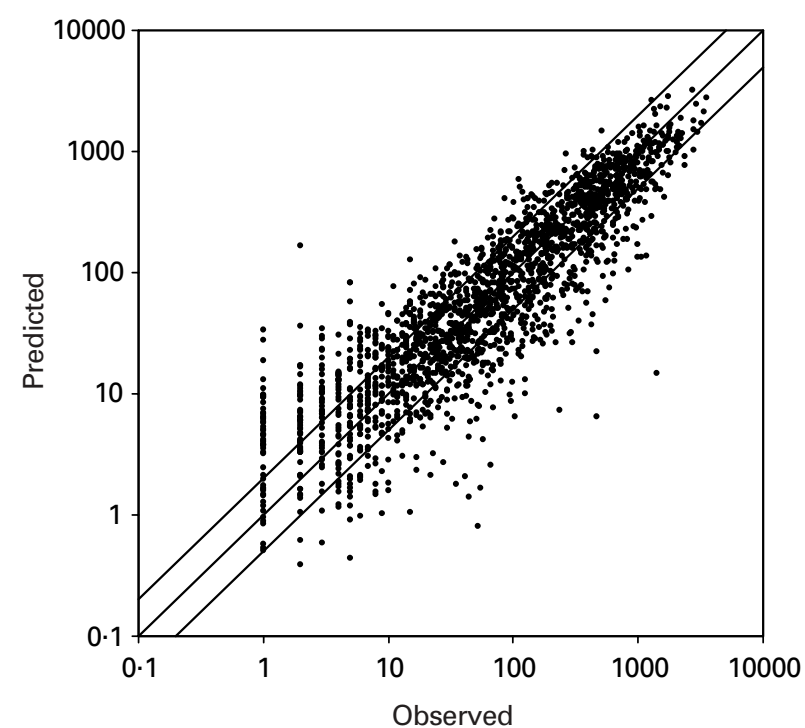

Fig. 2. Goodness-of-fit of the linear mixed model: observed $v s$ model-predicted values given in number of Plasmodium falciparum cases. Each dot represents a month and a site $(n=2067)$. The diagonal parallel lines indicate prediction values twice observed values (upper line), equal to observed values (middle line) and half the observed values (lower line). (Of the predictions, 19.7\% were twice the observed values and $18.6 \%$ were less than half of the observed values; furthermore, $10 \cdot 2 \%$ of all observations were grossly underestimated - i.e. greater than 200 cases per month per sector.)

high or low incidence $(>85 \%$ of the variance explained) than in those with moderate incidence (55-85\% of the variance explained).

The various extensions of the basic model are given in Table 3 with their respective likelihood ratio tests for goodness-of-fit in comparison to the basic model. In general, there was no significant improvement when maximum temperature was included. Due to the fact that rainfall relative to annual total in the previous month, and the quadratic terms of rainfall relative to annual total 2 months and 3 months earlier significantly improved the model, these factors were used in an extended model which improved the model significantly (Table 3 ). However, in terms of prediction and percentage under- or overestimated observations, virtually no improvement was obtained with the various extensions of the basic model, including the extended model.

In terms of percentage of observations grossly under-estimated ( $>200$ cases per month per sector), using the previous month's incidence as a prediction was surprisingly slightly better than the basic model $(9 \cdot 1 \%$ vs $10 \cdot 2 \%)$. However, in terms of percentage of ' not good enough' predictions, the basic model performed better $(38 \cdot 3 \%)$ than the model using previous month's incidence $(42.9 \%)$ (Table 4$)$. The alternative model based on the seasonal adjustment method was slightly worse than both the basic model and the previous month's incidence prediction (Table 4).

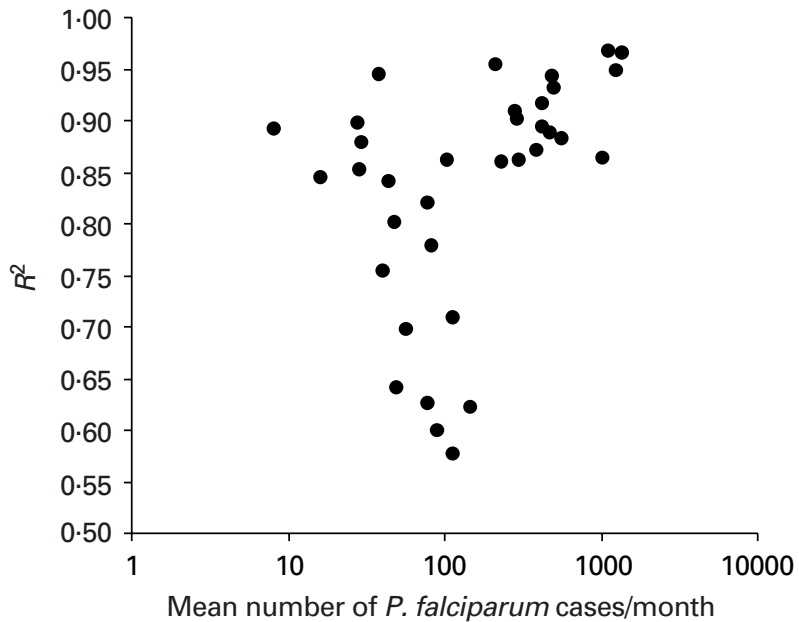

Fig. 3. Goodness-of-fit of the model (measured in $R^{2}$ values) as a function of mean monthly number of Plasmodium falciparum cases in each sector.

\section{DISCUSSION}

This study showed an association between monthly meteorological and malaria morbidity data in areas with unstable transmission using a statistical model based on theoretical reasoning. This linear mixed model, which includes rainfall 2 and 3 months earlier and mean minimum temperature in the previous month entered as fixed effects and incidence in previous month entered as a random effect, was fitted to malaria incidence data from 35 areas throughout Ethiopia. The model's fit was generally good especially in areas with high (and to some extent low) monthly incidence.

The model performed relatively poorly in areas with the mean number of cases per month between approximately 50 and 300 . This may be due to the fact that only in high and low endemicity areas the immunological status of the population is constant (high and low, respectively). These observations indicate a need to incorporate in a prediction model dynamic or temporally varying immunity levels. Although the model was motivated using immunological arguments and takes account of spatial variations in communal immunity levels across areas, it does not incorporate varying levels of immunity over time, to handle, for example, a consequence of recent outbreaks on incidence. Nevertheless, the developed theory of varying immunity is speculative and needs further study. It should be noted also that incidence and immunity levels interact in such a way that one leads to the other and models for epidemic early warning need to include such interactions. In an attempt to incorporate the level of immunity in forecasting incidence, the spleen rate has been used as a proxy to immunity status of the population in epidemic early warning in India, although this approach did not appear to help in providing an adequate basis for accurate forecast (Swaroop, 1949). In terms of 
Table 3. Comparison of goodness-of-fit of the basic model to its extensions by adding more predictor variables $(n=2067$ and $-2 \log$ likelihood of basic model $=5290 \cdot 1)$

\begin{tabular}{|c|c|c|c|c|}
\hline \multirow{2}{*}{$\begin{array}{l}\text { Additional factor } \\
\text { to the basic model }\end{array}$} & \multirow[b]{2}{*}{ Estimate } & \multirow[b]{2}{*}{ S.E. } & \multicolumn{2}{|c|}{$\begin{array}{l}\text { Test for significance of } \\
\text { improvement over basic model }\end{array}$} \\
\hline & & & $\chi_{1}^{2}$ & $P$ \\
\hline Minimum temperature 2 months earlier & $-0 \cdot 0047$ & $0 \cdot 0149$ & $9 \cdot 3$ & $0 \cdot 002$ \\
\hline Maximum temperature in the previous month & $0 \cdot 0048$ & $0 \cdot 0095$ & $0 \cdot 2$ & $0 \cdot 655$ \\
\hline Maximum temperature 2 months earlier & $0 \cdot 0080$ & $0 \cdot 0099$ & $0 \cdot 6$ & $0 \cdot 439$ \\
\hline Rainfall relative to annual total in the previous month & $2 \cdot 364$ & $0 \cdot 498$ & $22 \cdot 3$ & $<0 \cdot 001$ \\
\hline (Rainfall relative to annual total 2 months earlier) squared & $21 \cdot 913$ & $6 \cdot 240$ & $12 \cdot 2$ & $<0 \cdot 001$ \\
\hline (Rainfall relative to annual total 3 months earlier) squared & $20 \cdot 996$ & $6 \cdot 335$ & $10 \cdot 7$ & $0 \cdot 001$ \\
\hline
\end{tabular}

Table 4. Comparison of different models in terms of percentage of 'unacceptable' predictions (greater than twice or less than half observed values) and percentage of observations grossly underestimated (i.e. greater than 200 cases per month) $(n=2067)$

(The seasonal adjustment model was based on Abeku et al. (2002).)

\begin{tabular}{llll}
\hline \hline & $\begin{array}{l}\text { Predictions } \\
>\text { twice observed } \\
\text { values (\%) }\end{array}$ & $\begin{array}{l}\text { Predictions } \\
<\text { half observed } \\
\text { values }(\%)\end{array}$ & $\begin{array}{l}\text { Observations grossly } \\
\text { underestimated } \\
(>200 \text { cases per month) }(\%)\end{array}$ \\
\hline $\begin{array}{l}\text { Basic model (7) } \\
\begin{array}{l}\text { Extended model } \\
\text { Alternative model: current month's incidence } \\
\quad \text { previous month's incidence }\end{array}\end{array}$ & $\begin{array}{l}19 \cdot 7 \\
19 \cdot 2\end{array}$ & $18 \cdot 6$ & $10 \cdot 2$ \\
Alternative model: seasonal adjustment & $21 \cdot 0$ & $20 \cdot 9$ & $9 \cdot 7$ \\
\hline \hline
\end{tabular}

prediction, the present model, however, performed better than our best model that was devised previously based on historical incidence patterns alone (Abeku et al. 2002). This indicates that weather variables are essential components in a model used for epidemic prediction. Potentially confounding factors that affect transmission such as the level of drug resistant $P$. falciparum and the use of insecticides in malaria control in Ethiopia were ignored in the model, as (simple) prediction was our objective and the role of confounding factors was of less importance than in epidemiological studies of causation.

The $95 \% \mathrm{CI}$ for the estimates of the coefficient of incidence in the previous month $(0 \cdot 69-0 \cdot 75)$ indicated a uniform value for most areas in the country. This is in concordance with the fact that most sectors in Ethiopia have similar endemicity levels. The individual effect of each of the predictor variables was investigated in the present study using model estimates for which the best fit was obtained, while keeping the other variables constant. Increased minimum temperature resulted in increase in incidence up to a threshold limit of approximately $23{ }^{\circ} \mathrm{C}$, after which increase in minimum temperature ceased to have an effect on incidence. Around $14^{\circ} \mathrm{C}$, an increase in minimum temperature of $1{ }^{\circ} \mathrm{C}$ resulted in $8 \%$ increase in incidence. The exponential effect of rainfall associates with a $3 \%$ increase in incidence for every $1 \%$ increase in rainfall.
Although more detailed studies are still required to thoroughly understand the impact of meteorological variables in the genesis of epidemics in different areas, it seems that the effect of rainfall varies from sector to sector depending on prevailing temperature and other epidemiological factors. Previously, an inverse relationship was found between rainfall and incidence in southern Ethiopia in drought-associated epidemics due to breeding of vector mosquitoes in pools formed in river beds and streams (unpublished data). Abnormally high rainfall is a causative factor for epidemics in lowlands and fringe areas bordering lowlands. In the cooler highlands, temperature (especially minimum temperature) has a more profound role in determining malaria transmission. A drop in temperature has been shown to be associated with interruption of transmission in the highland sectors of Ethiopia (Abeku et al. 2003). In the hypoendemic highlands, temperature exerts its effect on transmission mainly as the result of shortened sporogonic cycle of the parasite within the vector, and to some extent also by accelerating larval development and frequency of blood feeding by the vector.

In a study conducted in Rwanda, Loevinsohn (1994) showed that changes in malaria incidence at health facilities were significantly associated with rainfall while temperature predicted incidence best at higher altitudes. It was shown that a model that included $\log$ of minimum temperature 1 and 2 months 
earlier, and rainfall 2 and 3 months earlier fitted the health facility data adequately. In our study minimum temperature in the previous month included with its quadratic term usually gave adequate fits in the presence of the previous month's incidence level. Incidence was not included as a predictor variable in the Rwandan study, but in our model we have shown that it is highly significant as a determinant of incidence in the following month. Previously, we have shown that malaria epidemics in Ethiopia were significantly more often preceded by a month of abnormally high minimum temperature in the preceding 3 months than based on random chance (Abeku et al. 2003). In another observation made in Zimbabwe, Freeman \& Bradley (1996), reported that rainfall has little effect on severity of malaria (assessed by comparing the numbers of malaria deaths and malaria-inpatients in any one year with respect to those in the preceding years), while temperature has an effect. In Uganda, Kilian et al. (1999) reported the existence of a close correlation between peak of rainfall and peak of malaria incidence with a time lag of 2-3 months between them. In a study conducted in central Ethiopia, Tulu (1996) reported that a rise in monthly mean minimum temperatures 2 and 3 months earlier was the strongest predictor of a rise in incidence. In the present study, the inclusion of minimum temperature of 2 or 3 months earlier did not improve the basic model and the effects were not significant whereas the effect of minimum temperature of the previous month (already in the model) was strongly significant.

To test whether changes in the weather variables have different effects on incidence in highlands and lowlands, we carried out a stratified analysis by dichotomizing altitude into high $(>1750 \mathrm{~m})$ and low $(\leqslant 1750 \mathrm{~m})$ and including in the model interaction terms between meteorological and the dichotomized altitude variables. The altitude variable and all the interactions with the weather variables did not have significant coefficients, and the inclusions did not improve the basic model; the main results of the study remained unchanged. This is probably due to either absence of difference in effects of meteorological variables at different altitudes or due to the already included temperature variables in the model as temperature is strongly negatively correlated with altitude, thus indirectly taking account of the effect of altitude.

Climate-based distribution of malaria transmission and infection risk models for Africa has been proposed (Craig, Snow \& Le Sueur, 1999) and spatially predictive models have been prepared for epidemicaffected areas of Africa (Cox et al. 1999). The present study has indicated how the association between some meteorological factors and incidence may be modeled in a continuing effort to develop epidemic early warning systems in highland areas for temporal prediction.
Satellite-derived remote sensing (RS) data are potentially useful for monitoring malaria epidemics, although in some cases they may not provide accurate spatial proxies to actual ground meteorological measurements. The relative accuracy of RS and spatial interpolation (SI) of data from meteorological stations has been assessed for the prediction of spatial variation in monthly climate across Africa (Hay \& Lennon, 1999). It has been found that SI was a more accurate predictor of temperature, whereas RS provided a better surrogate for rainfall. On the other hand, it has been shown that Normalized Difference Vegetation Index (NDVI) in the previous month correlated consistently with malaria incidence across three sites in Kenya (Hay et al. 1998). Although there is obviously no direct causal link between NDVI and malaria cases to use it as a variable in the current model, the relationships between this and other RS data, ground meteorological records and malaria incidence in the highlands need to be further investigated, for possible use in similar models. A detailed study has been initiated to investigate such relationships with epidemic malaria in four highland districts in Kenya and Uganda, as part of the Highland Malaria Project (HIMAL) (www.himal.uk.net).

The present analysis showed that a statistical model based on theoretical reasoning is a good starting point to understand the role of abnormal weather variables in triggering epidemics in the highlands or highland fringe areas, and that the impact of the effects of these variables in terms of morbidity outcomes may depend on several factors including communal immunity and number of pre-epidemic parasite reservoirs in the population.

Prediction of incidence several months in advance will require major adaptation of the current model, for example, by making use of predicted values of the predictor variables themselves. However, it is anticipated that the accuracy of such prediction would deteriorate after a few months, compared to the (already moderate) performance of one month prediction. Further validation is also needed by fitting the model to new data sets to estimate random effects by using optimal estimates of the fixed parameters. Ways to improve forecasts by making use of past patterns of incidence and other variables and/or by combining seasonality and weighted forecasts of different methods in relation to population immunity levels are currently under investigation. In terms of prediction of malaria incidence using the basic model, although the main contribution comes from the previous month's incidence, the weather parameters included are highly significant and values of their coefficients meet our expectations. Also, we have demonstrated that inclusion of maximum temperature is not important at all. Nevertheless, the study shows that prediction rules derived from simple and straightforward use of monthly weather variables alone might not produce accurate forecasts. In 
addition, it may be important to study the weathermalaria relationships in some more details using time series of weather, morbidity and entomological variables at intervals of less than a month. The modelling approach used in this study has shown the most important variables that need to be considered in developing a malaria epidemic early warning system in areas where communities are at risk of sudden increase in transmission due to slight changes in the precipitating factors.

We are grateful to the National Meteorological Services Agency and the Ministry of Health of the Federal Democratic Republic of Ethiopia for providing data used for the analyses and for facilitating the study. Financial support for this study was provided by The Trust Fund, Erasmus University Rotterdam, The Netherlands Institute of Health Sciences (NIHES), Gates Malaria Partnership and DfID Malaria Knowledge Programme. S.J.V. received financial support from the Netherlands Foundation for the Advancement of Tropical Research (WOTRO/NWO). Compilation of meteorological data was supported by the United Nations Development Programme (UNDP).

\section{REFERENCES}

ABEKU, T. A., DE Vlas, S. J., BORSBOOM, G., TEKLEHAimanoT, A., Kebede, A., Olana, D., VAN OORTMARSSEN, G. J. \& HABBEMA, J. D. F. (2002). Forecasting malaria incidence from historical morbidity patterns in epidemic-prone areas of Ethiopia: a simple seasonal adjustment method performs best. Tropical Medicine and International Health 7, 851-857.

ABEKU, T. A., VAN OORTMARSSEN, G. J., BORsboom, G., DE VLAS, S. J. \& HABBEMA, J. D. F. (2003). Spatial and temporal variations of malaria epidemic risk in Ethiopia: factors involved and implications. Acta Tropica 87, 331-340.

BOUMA, M. J. \& DYE, C. (1997). Cycles of malaria associated with El Niño in Venezuela. Fournal of the American Medical Association 278, 1772-1774.

Bouma, M. J. \& VAN DER KAAY, J. (1996). The El Niño Southern Oscillation and the historic malaria epidemics on the Indian subcontinent and Sri Lanka: an early warning system for future epidemics? Tropical Medicine and International Health 1, 86-96.

COX, J., CRAIG, M., LE SUEUR, D. \& SHARP, B. (1999). Mapping Malaria Risk in the Highlands of Africa. MARA/ HIMAL Technical Report.

CRAIG, M. H., SNOW, R. W. \& LE SUEUR, D. (1999). A climatebased distribution model of malaria transmission in Sub-Saharan Africa. Parasitology Today 15, 105-111.

FONTAINE, R. E., NAJJAR, A. E. \& PRINCE, J.s. (1961). The 1958 malaria epidemic in Ethiopia. American Fournal of Tropical Medicine and Hygiene 10, 795-803.

FREEMAN, T. \& BRADLEY, M. (1996). Temperature is predictive of severe malaria years in Zimbabwe.
Transactions of the Royal Society of Tropical Medicine and Hygiene 90, 232.

HAY, S. I. \& LENNON, J. J. (1999). Deriving meteorological variables across Africa for the study and control of vector-borne diseases: a comparison of remote sensing and spatial interpolation of climate. Tropical Medicine and International Health 4, 58-71.

HAY, S. I., OMUMBO, J. A., CRAIG, M. H. \& SNOW, R. W. (2000). Earth observation, geographic information systems and Plasmodium falciparum malaria in Sub-Saharan Africa. Advances in Parasitology 47, 173-215.

HAY, S. I., SNOW, R. W. \& ROGERS, D. J. (1998). Predicting malaria seasons in Kenya using multitemporal satellite sensor data. Transactions of the Royal Society of Tropical Medicine and Hygiene 92, 12-20.

Kilian, A. H. D., LANGi, P., TAlisuna, A. \& Kabagambe, G. (1999). Rainfall pattern, El Niño and malaria in Uganda. Transactions of the Royal Society of Tropical Medicine and Hygiene 93, 22-23.

LindSAy, s. W. \& MARTENS, W. J. M. (1998). Malaria in the African highlands: past, present and future. Bulletin of the World Health Organization 76, 33-45.

Loevinsohn, M. E. (1994). Climate warming and increased malaria incidence in Rwanda. The Lancet 343, 714-718.

MYERS, M. F., ROGERS, D. J., COX, J., FlaHAUlT, A. \& HAY, S. (2000). Forecasting disease risk for increased epidemic preparedness in public health. Advances in Parasitology 47, 309-330.

Macdonald, G. (1957). The Epidemiology and Control of Malaria. Oxford: Oxford University Press.

MOLineaux, L. (1988). Malaria: the epidemiology of human malaria as an explanation of its distribution, including some implications for control. In Principle and Practice of Malariology (ed. Wernsdorfer, W. H. \& McGregor, I.), pp. 913-998. Churchill Livingstone, Edinburgh.

NÁJERA, J. A., Kouznetzsov, R. L. \& DELACOLleTte, C. (1998). Malaria Epidemics: Detection and Control, Forecasting and Prevention. World Health Organization document: WHO/MAL/98.1084.

SAS INSTITUTE INC. (1999). SAS/STAT ${ }^{\mathrm{R}}$ User's Guide, Version 8. Cary, NC: SAS Institute Inc., USA.

SWAROOP, s. (1949). Forecasting of epidemic malaria in the Punjab, India. American Fournal of Tropical Medicine 29, 1-17.

THOMson, M. C. \& CONNOR, s. J. (2001). The development of malaria early warning systems for Africa. Trends in Parasitology 17, 438-445.

TUlu, A. N. (1996). Determinants of malaria transmission in the highlands of Ethiopia: The impact of global warming on morbidity and mortality ascribed to malaria. $\mathrm{Ph} . \mathrm{D}$. thesis. London School of Hygiene and Tropical Medicine, University of London.

verbeke, G. \& Molenberghs, G. (2000). Linear Mixed Models for Longitudinal Data. New York, Springer. 